\title{
Análisis de la restauración pasiva post-agrícola de Prosopis laevigata en el matorral espinoso Tamaulipeco
}

\section{Analysis of post-agricultural passive restoration of Prosopis laevigata in Tamaulipan thornscrub}

Miguel Ángel Pequeño-Ledezma 1 [D Eduardo Alanís-Rodríguez ${ }^{1 *}$ (D) Laura Sánchez-Castillo 1 [D Arturo Mora-Olivo2 2

Ernesto Alonso Rubio-Camacho ${ }^{3}$ (D) E. Alejandro Lozano-Cavazos ${ }^{4}$ (D)

${ }^{1}$ Facultad de Ciencias Forestales, Universidad Autónoma de Nuevo León, Apartado Postal 41, CP. 67700. Linares, Nuevo León, México.

${ }^{2}$ Instituto de Ecología Aplicada, Universidad Autónoma de Tamaulipas, Matamoros s/n, Zona Centro CP. 87000 Ciudad Victoria, Tamaulipas, México.

${ }^{3}$ INIFAP-CIRPAC, C.E. Km.

8

Carretera Libre Tepatitlán-Lagos de Moreno Av. Biodiversidad \#2470, CP. 47600 Tepatitlán de Morelos, Jalisco, México.

${ }^{4}$ Departamento de Recursos Naturales Renovables, Universidad Autónoma Agraria Antonio Narro, Calzada Antonio Narro \#1923, Buenavista, CP. 25315 Saltillo, Coahuila, México.

*Autor de correspondencia:

eduardoforestal@gmail.com

\section{Artículo científico}

Recibido: 30 de abril de 2019

Aceptado: 17 de octubre de 2019

Como citar: Pequeño-Ledezma MA, Alanís-Rodríguez E, SánchezCastillo L, Mora-Olivo A, RubioCamacho EA, Lozano-Cavazos EA (2020) Análisis de la restauración pasiva post-agrícola de Prosopis laevigata en el matorral espinoso Tamaulipeco. Ecosistemas y Recursos Agropecuarios 7(1): e2247. DOI: 10.19136/era.a7n1.2247
RESUMEN. El área cubierta por matorral representa la mayor superficie por tipo de vegetación de México, las prácticas agropecuarias tienden a estar presentes abarcando un amplio territorio. El objetivo fue analizar la regeneración de las especies leñosas post-actividad agrícola en un área de matorral espinoso tamaulipeco en el noreste de México perteneciente al Rancho San Felipe, ubicado en el municipio de General Terán, Nuevo León. Se establecieron seis sitios de muestreo de $10 \times 20$ $\mathrm{m}$ para tener una superficie total de $1200 \mathrm{~m}^{2}$, en los que se realizó un inventario de especies leñosas. Para su análisis se utilizaron los indicadores ecológicos de abundancia, dominancia, frecuencia, índice de valor de importancia por especie y de manera general los índices de Margalef, Shannon y Wiener, y el índice de Pretzsch. La especie dominante, en cobertura fue Prosopis laevigata $\left(4731.1 \mathrm{~m}^{2}\right)$, que representa el $89.12 \%$, seguida de Acacia farnesiana con $9.66 \%$ y Celtis pallida con $1.22 \%$ del total de los individuos. Se registró una abundancia absoluta de 733 $\mathrm{N}$ ha ${ }^{-1}$, donde Prosopis laevigata representó el $84.09 \%$ del total. De acuerdo a la distribución diamétrica, la mayoría de los individuos (350 $\left.\mathrm{N} \mathrm{ha}^{-1}\right)$ se encuentran en el rango de 0 a $5 \mathrm{~cm}$ de diámetro, lo que indica que se está regenerado activamente. En cuanto a la distribución vertical se observó que la vegetación presenta una diversidad media en los estratos de altura, registrando un valor de $63.7 \%$ de $\mathrm{A}_{\text {rel }}$. La comunidad vegetal muestra un estado de regeneración activo.

Palabras clave: Abundancia, agricultura, densidad, dominancia, regeneración.

ABSTRACT. The thornscrub ecosystem is the most distributed in Mexico and historically has been used for agricultural purposes in arid and semiarid regions. The objective of this research was to analyze the regeneration of woody species post-agricultural activity in an area of Tamaulipas thorny thicket in northeastern Mexico belonging to Rancho San Felipe, located in the municipality of General Terán, Nuevo León. Six sampling sites of $10 \times 20$ m were established to have a total area of $1200 \mathrm{~m}^{2}$ A shrub species census was carried out at sampling sites. We used ecological indicators such as abundance, dominance, frequency, importance value index, Margalef index, index of Shannon and Wiener, and Pretzsch index. The dominant specie in the study area is Prosopis laevigata, which represents $89.12 \%$, followed by Acacia farnesiana with $9.66 \%$ and Celtis pallida with $1.22 \%$ of the total of the individuals. The absolute abundance of 733 individuals was recorded, where Prosopis laevigata represented $84.09 \%$ of the total. According to the diametric distribution, the majority of the individuals $\left(350 \mathrm{~N} \mathrm{ha}^{-1}\right)$ were in the range of 0 to 5 $\mathrm{cm}$ in diameter, which indicates an active regeneration of the ecosystem. Regarding to the vertical distribution, it was observed that the vegetation presents a medium diversity in the height strata, recording a value of $63.7 \%$ of $A_{\text {rel }}$. The regenerated plant community shows a state of active regeneration.

Key words: Abundance, agriculture, density, dominance, regeneration. 


\section{INTRODUCCIÓN}

El sector agrícola representa el 3.8\% del Producto Interno Bruto en el mundo (Banco Mundial 2016), cerca del $29 \%$ de la superficie terrestre se encuentra ocupada por pastizales permanentes o bien por cultivos forrajeros utilizados para la cría de ganado (Fresco 2005). Al respecto McMahon y Valdés (2011) mencionan que la agricultura es un sector pequeño en México, que se encuentra a la baja con respecto a la economía total, representando cerca del $4 \%$ del PIB. De acuerdo con la encuesta nacional agropecuaria 2014 la superficie agrícola en México es de 109.3 millones de hectáreas, de las que se utiliza el $25.2 \%$, que equivalen a 27.5 millones de hectáreas, de las que el $81.5 \%$ corresponde a superficie sembrada o plantada. Mientras que las 81.8 millones de hectáreas restantes corresponden a superficie de agostadero, enmontada o en descanso (SAGARPA-INEGI 2015). Particularmente, el estado de Nuevo León cuenta con una superficie agrícola que asciende a 392415 hectáreas, que representan casi el $2 \%$ de la superficie agrícola del país, de las cuales el $67 \%$ son de temporal y el $33 \%$ de riego (SAGARPA 2009). El desarrollo agrícola, en conjunto con otras actividades humanas como la industria y la urbanización, han contribuido a la deforestación de diversos tipos de vegetación como el caso de los matorrales (Alanís et al. 2008, Arriaga 2009). Estas comunidades se consideran las más abundantes y las más utilizadas en las zonas áridas y semiáridas de México (García y Jurado 2008). Entre 1993 y 2002 el matorral sufrió una pérdida de 953000 ha por cambio de uso de suelo, siendo el segundo ecosistema más afectado después de las selvas (SEMARNAT 2006). A pesar de lo anterior, se conoce que bajo ciertas circunstancias la sola suspensión de actividades humanas dañinas para un ecosistema, puede generar las condiciones básicas para la restauración en forma autónoma (Zahawi et al. 2015, Meli et al. 2017). Esta restauración pasiva es favorecida si la extensión del daño es pequeña, si no existe secuela alguna de contaminación presente y si existen áreas aledañas que cuenten con germoplasma nativo local
(Bajaña et al. 2014). Por lo que las áreas destinadas a actividades productivas utilizadas durante un cierto tiempo y abandonadas cuando bajan su productividad, pueden regenerarse de forma natural (restauración pasiva). Pero hay evidencia de que en escalas espaciales y temporales amplias la restauración es afectada por la diversidad y riqueza de arbustivas con respecto a sitios no disturbados (Fulbright et al. 2013).

Actualmente existen comunidades vegetales escasamente evaluadas desde el punto de vista de la regeneración pasiva, como el matorral espinoso tamaulipeco (MET) (Pequeño-Ledezma et al. 2012). Por esta razón, es pertinente evaluar con detalle los ecosistemas resultantes de las perturbaciones causadas por la agricultura (Jiménez et al. 2012, Alanís et al. 2015). Ello genera conocimiento ecológico cuantitativo útil para la gestión y conservación de los ecosistemas, ya que la información producida es la base para desarrollar proyectos de restauración ecológica (Pequeño-Ledezma et al. 2016). Consecuentemente, el objetivo fue analizar la regeneración de las especies leñosas post-actividad agrícola en un área de matorral espinoso tamaulipeco en el noreste de México perteneciente al Rancho San Felipe, ubicado en el municipio de General Terán, Nuevo León.

\section{MATERIALES Y MÉTODOS}

\section{Área de estudio}

La investigación se realizó en una fracción del matorral espinoso tamaulipeco en el municipio de General Terán, Nuevo León, México, situada a $25^{\circ} 6^{\prime} 54.48^{\prime \prime}$ LN, y 99 12 '56.47" LO, Que tiene una superficie de 473 ha a $209 \mathrm{msnm}$. En específico el área de estudio se localizó en el Rancho San Felipe, ubicado en el municipio de General Terán, Nuevo León (Figura 1). Los sitios seleccionados se encuentran dentro de un área de matorral espinoso tamaulipeco con historial agrícola, que tiene un abandono de 15 años. Esta área fue desmontada con maquinaria agrícola, lo que implicó remoción de suelo, y se utilizó por cinco años para el cultivo de maíz y sorgo de temporal. 


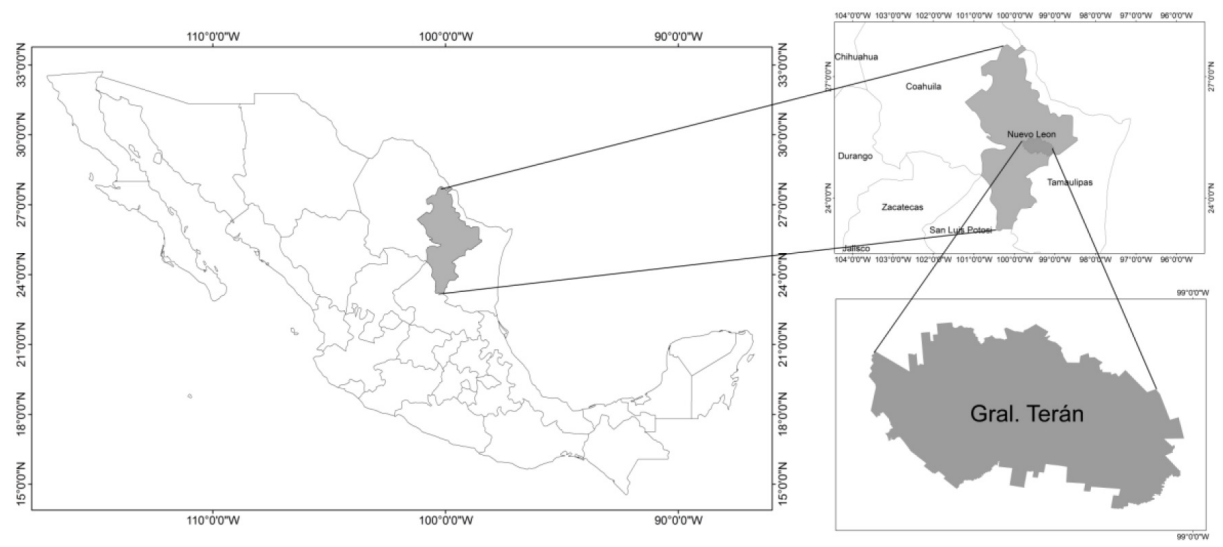

Figura 1. Localización del municipio de General Terán.

\section{Inventario florístico}

En el verano del 2014, 15 años después del disturbio ocasionado por la actividad agrícola que se realizaba en la zona, se establecieron seis sitios de muestreo para evaluar la regeneración de la vegetación leñosa. Los sitios de muestreo fueron de $10 \times 20 \mathrm{~m}\left(200 \mathrm{~m}^{2}\right)$, para tener una superficie total de $1200 \mathrm{~m}^{2}$; en cada sitio se realizó un censo de las especies leñosas, a las que se les midió la altura total $(h)$, diámetro de copa $(d)$ y diámetro basal $(D b)$. El diámetro se determinó a $0.10 \mathrm{~m}$ sobre la base del suelo, ya que es una medida estándar empleada para la regeneración de las especies leñosas del MET (Alanís et al. 2008).

\section{Análisis de la información}

Para cada especie se determinó su abundancia, de acuerdo con el número de árboles, cobertura en función del área de copa, y su frecuencia con base en su presencia en los sitios de muestreo. Las variables relativizadas se utilizaron para obtener un valor ponderado a nivel de taxón denominado Índice de valor de importancia (IVI), que adquiere valores porcentuales en una escala de 0 a 100 (Mostacedo y Fredericksen 2000). Para la estimación de la abundancia relativa se empleó la siguiente fórmula:

$$
\begin{gathered}
A R_{i}=\left(A_{i} / \sum A_{i}\right) * 100 \\
\mathrm{i}=1 \ldots \mathrm{n}
\end{gathered}
$$

Dónde: $\mathrm{AR}_{i}$ es la abundancia relativa de la especie i respecto a la abundancia total y Ai es la abundancia absoluta de la especie i (N/ha). La dominancia se determinó con la fórmula:

$$
\begin{gathered}
D R_{i}=\left(D_{i} / \sum D_{i}\right) * 100 \\
\mathrm{i}=1 \ldots \mathrm{n}
\end{gathered}
$$

Dónde: DRi es la dominancia relativa de la especie i respecto a la dominancia total y $D$ es la dominancia absoluta de la especie $\mathrm{i}\left(\mathrm{m}^{2} / \mathrm{ha}\right)$. Las frecuencias absoluta y relativa se obtuvieron con las fórmulas:

$$
\begin{gathered}
F_{i}=\left(f_{i} / N\right) * 100 \\
F R_{i}=\left(F_{i} / \sum F_{i}\right) * 100 \\
\mathrm{i}=1 \ldots \mathrm{n}
\end{gathered}
$$

Dónde: $F_{i}$ es la frecuencia absoluta (porcentaje de presencia en los sitios de muestreo), $f_{i}$ es el número de sitios en la que está presente la especie $i, N$ es el número de sitios de muestreo y $F R_{i}$ es la frecuencia relativa de la especie i respecto a la frecuencia total. El índice de valor de importancia (IVI) se determinó con la fórmula:

$$
I V I=\frac{A R_{i}+D R_{i}+F R_{i}}{3}
$$

Para estimar la diversidad alfa se utilizó el índice de Margalef (DMg) y el índice de Shannon \& Weiner ( $\left.\mathrm{H}^{\prime}\right)$, con las fórmulas:

$$
D_{M g}=\frac{(s-1)}{\ln (N)}
$$




$$
\begin{gathered}
H^{\prime}=-\sum_{i=1}^{S} p_{i} * \ln \left(p_{i}\right) \\
p_{i}=n_{i} / N
\end{gathered}
$$

Dónde: $S$ es el número de especies presentes, $N$ es el número total de individuos y ni es el número de individuos de la especie $i$.

Para la caracterización de la estructura vertical de las especies se utilizó el índice de distribución vertical de especies (A) (Pretzsch 2009, Del Río et al. 2003). Donde: $A$ indica valores entre 0 y un valor máximo $\left(A_{\max }\right)$. Un valor $A=0$ significa que el rodal está constituido por una sola especie que ocurre en un solo estrato. $A_{\max }$ se alcanza cuando la totalidad de las especies ocurren en la misma proporción tanto en el rodal como en los diferentes estratos (Pretzsch 2009, Corral et al. 2005). Para la estimación de la distribución vertical de las especies, se definieron tres zonas de altura (Pretzsch 2009, Jiménez et al. 2001), siendo éstas: zona I: 80 a $100 \%$ de la altura máxima del área, zona II: 50 a $80 \%$, y zona III: de 0 a 50\%. Este índice (A) sirve para determinar la diversidad estructural en cuanto a la distribución vertical de las especies y se calcula con la siguiente fórmula:

$$
A=-\sum_{i=1}^{S} \sum_{i=1}^{Z} p_{i j} * \ln \left(p_{i j}\right)
$$

Dónde: $\mathrm{S}=$ número de especies presentes; $\mathbf{Z}=$ número de estratos de altura; $p_{i j}=$ porcentaje de especies en cada zona, y se estima mediante la siguiente fórmula:

$$
p_{i j}=n_{i, j} / N(10)
$$

Dónde: $\mathrm{n}_{i, j}=$ número de individuos de la misma especie (i) en la zona (j) y $\mathrm{N}=$ número total de individuos.

Para poder comparar el índice de Pretzsch es necesario estandarizarlo, lo que se realizó mediante el valor de $\mathrm{A}_{m x}$, que se calcula de la siguiente manera:

$$
\mathbf{A}_{\max }=\ln (S X Z)(11)
$$

Entonces se puede estandarizar el valor de $\mathrm{A}$ acorde a:

$$
A_{\text {rel }}=\frac{A}{\ln (S * Z)} * 100
$$

\section{RESULTADOS}

Se registraron tres especies pertenecientes a dos familias de plantas vasculares. La familia más abundante fue Fabaceae con dos especies, la familia restante presentó solo una especie. Las formas biológicas de las especies presentes fueron arbórea y arbustiva (Tabla 1). El patrón de abundancia relativa muestra que la especie más abundante es Prosopis laevigata, con un valor de $84 \%\left(616 \mathrm{~N} \mathrm{ha}^{-1}\right)$ del total de los individuos evaluados. El segundo y tercer lugar lo tuvieron Acacia farnesiana y Celtis pallida que representaron 14 y $1 \%$, respectivamente.

La comunidad evaluada tuvo una cobertura de copa de $5309 \mathrm{~m}^{2} \mathrm{ha}^{-1}$, lo que indica una cobertura del $53 \%$. La especie dominante fue Prosopis laevigata con un $89.12 \%$, seguida de Acacia farnesiana y Celtis pallida con 9.66 y $1.22 \%$, respectivamente del total de los individuos. La especie de mayor frecuencia relativa en el área de estudio fue Prosopis laevigata, con presencia en todos los sitios de muestreo. Acacia farnesiana tuvo un valor de $36.36 \%\left(\mathrm{~N} \mathrm{ha}^{-1}\right)$ y Celtis pallida de $9.09 \%\left(\mathrm{~N} \mathrm{ha}^{-1}\right)$. Prosopis laevigata y Acacia farnesiana fueron las especies que registraron los valores más altos en abundancia, dominancia y frecuencia, siendo Prosopis laevigata la de mayor valor de importancia con $75.92 \%$ (Tabla 2).

En la Figura 2 se observa una línea de tendencia exponencial negativa en la densidad de individuos conforme aumenta el diámetro de los mismos, siendo la clase $0-5 \mathrm{~cm}$ de diámetro la que presentó valores superiores a los $350 \mathrm{~N} \mathrm{ha}^{-1}$. Mientras que en la Figura 3 se observa la abundancia de individuos por hectárea de Prosopis laveigata, la cual es dominante en el área de estudio. Se aprecia también una línea de tendencia exponencial negativa, con decremento de abundancia con el aumento de las categorías diamétricas, siendo las clases < 5 y 5 - 10 las que presentan la abundancia más alta con valores de 267 
Tabla 1. Nombre científico, común, familia y forma biológica de las especies presentes en el área.

\begin{tabular}{lccc}
\hline Nombre científico & Nombre común & Familia & Forma biológica \\
\hline Prosopis laevigata (Humb. \& Bonpl. ex Willd.) M.C. Johnst. & Mezquite & Fabaceae & Arbórea \\
Acacia farnesiana (L.) Willd. & Huizache & Fabaceae & Arbórea \\
Celtis pallida Torr. & Granjeno & Ulmaceae & Arbustiva \\
\hline
\end{tabular}

Tabla 2. Abundancia ( $\mathrm{N} / \mathrm{ha})$, dominancia $\left(\mathrm{m}^{2} / \mathrm{ha}\right)$, frecuencia e índice de Valor de importancia de las especies registradas (ordenados decrecientemente de acuerdo a su IVI).

\begin{tabular}{lccccccc}
\hline & \multicolumn{2}{c}{ Abundancia } & \multicolumn{3}{c}{ Dominancia } & \multicolumn{2}{c}{ Frecuencia } \\
Nombre Científico & $\mathrm{N} / \mathrm{ha}$ & $\mathrm{Ar}$ & $\mathrm{m}^{2} / \mathrm{ha}$ & $\mathrm{D}_{r}$ & $\mathrm{Abs}$ & $\mathrm{Rel}$ & $\mathrm{IVI}$ \\
\hline Prosopis laevigata & 616 & 84.09 & 4731.1 & 89.12 & 100 & 54.55 & 75.92 \\
Acacia farnesiana & 108 & 14.77 & 512.61 & 9.66 & 66.67 & 36.36 & 20.26 \\
Celtis pallida & 8 & 1.14 & 64.91 & 1.22 & 16.67 & 9.09 & 3.82 \\
\hline
\end{tabular}

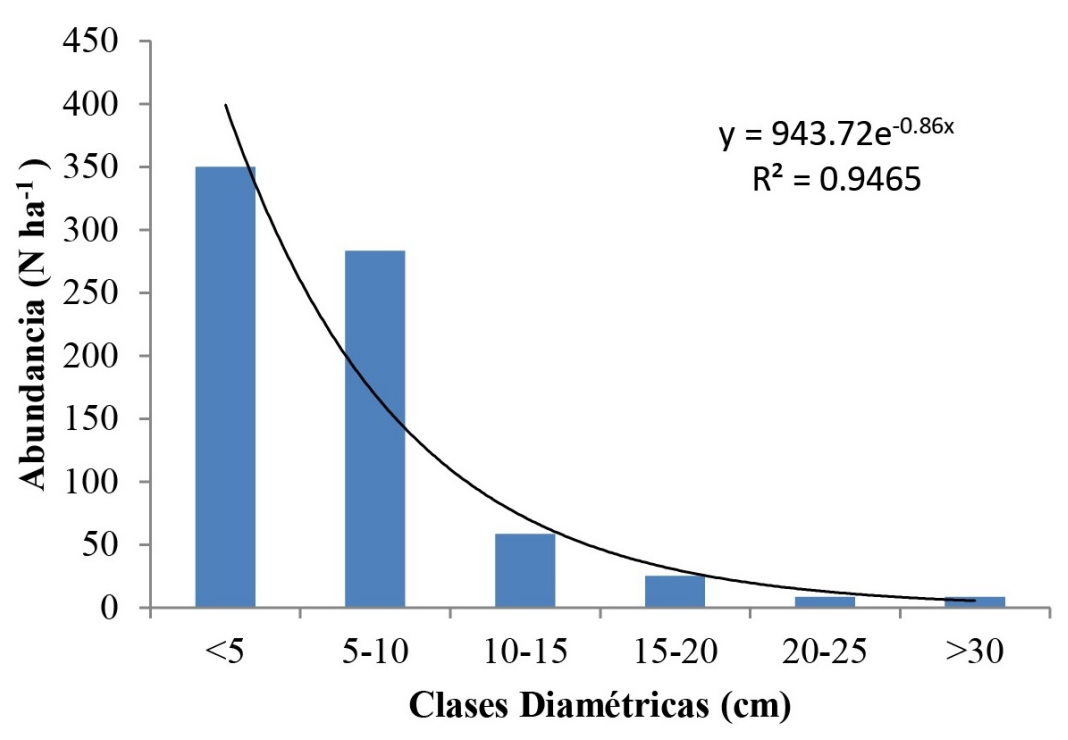

Figura 2. Abundancia de los individuos de acuerdo a clases diamétricas en el área de estudio.

$\mathrm{N}$ ha ${ }^{-1}$ para cada una de las categorías diamétricas. En la Figura 4 se muestra la abundancia de los individuos registrados en el área de estudio de acuerdo a las clases de altura. Se observa que existe una distribución normal, donde los árboles de dimensiones bajas y altas son poco abundantes y los árboles de altura intermedia $(2.5-3.0 \mathrm{~m})$ presentan la mayor abundancia.

\section{Riqueza y diversidad}

El área evaluada mostró valores de riqueza $\mathrm{D}_{M g}=0.45$ y diversidad alfa de $\mathrm{H}^{\prime}=0.48$. El valor obtenido del índice vertical de especies (A) fue 1.4 con un $A_{\text {max }}$ de 2.2 y un $A_{\text {rel }}$ de $63.7 \%$, lo que indica diversidad estructural media en los estratos de altura. La Tabla 3 muestra que existe una presencia alta del estrato II, con un $57.98 \%$ de los individuos. El análisis de distribución vertical con los estratos, alto, medio y bajo; indica que el estrato alto está formado por Prosopis laevigata, Celtis pallida y Acacia farnesiana con 167, 8 y $17 \mathrm{~N} \mathrm{ha}^{-1}$, respectivamente. En tanto que el estrato medio lo forman las especies Prosopis laevigata y Acacia farnesiana con 376 y 58 $\mathrm{N}$ ha ${ }^{-1}$ que representan el 86 y $14 \%$ de las especies, respectivamente (Tabla 3). Para el estrato bajo se encontraron estas mismas dos especies siendo la más abundante Prosopis laevigata con $83 \mathrm{~N} \mathrm{ha}^{-1}$, seguida Acacia farnesiana con $33 \mathrm{~N} \mathrm{ha}^{-1}$ (Tabla 3 ). 


\section{Prosopis laevigata}

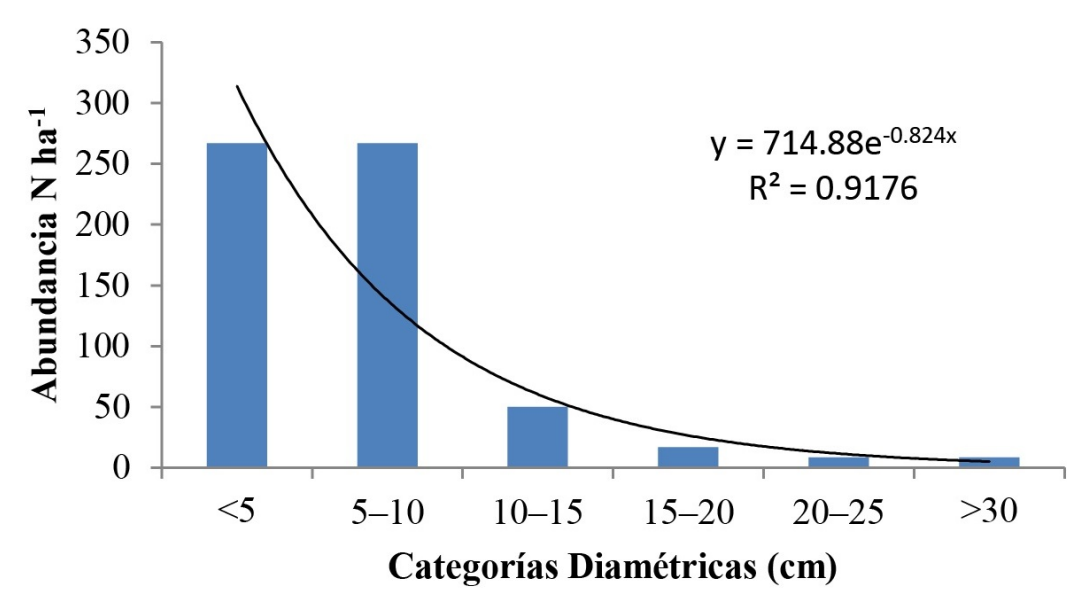

Figura 3. Abundancia de Prosopis laevigata de acuerdo a las clases diamétricas.

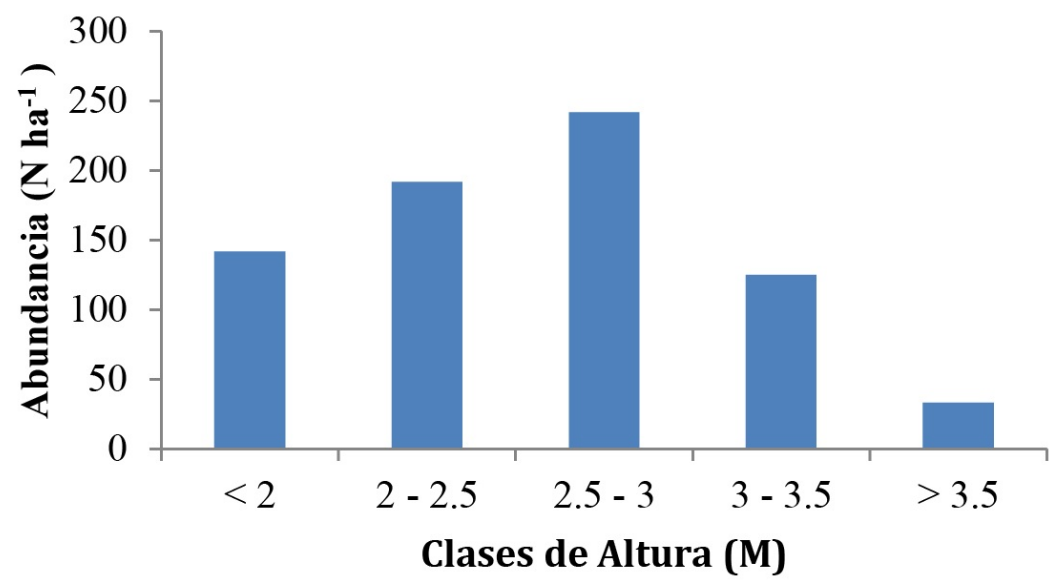

Figura 4. Abundancia de los individuos de acuerdo a las clases de altura.

Tabla 3. Valores del índice vertical de Pretzsch para el matorral espinoso tamaulipeco del área de estudio.

\begin{tabular}{|c|c|c|c|c|c|}
\hline \multirow[b]{2}{*}{ Estrato } & \multirow[b]{2}{*}{ Nombre científico } & \multirow[b]{2}{*}{$\mathrm{N}$} & \multirow[b]{2}{*}{$\mathrm{N} \mathrm{ha}^{-1}$} & \multicolumn{2}{|c|}{$\begin{array}{l}\text { Índice de Pretzsch } \\
\text { Proporción (\%) }\end{array}$} \\
\hline & & & & Del total & En la zona \\
\hline & Prosopis laevigata & 20 & 167 & 86.96 & 22.74 \\
\hline \multirow[t]{4}{*}{ I } & Celtis pallida & 1 & 8 & 4.35 & 1.14 \\
\hline & Acacia farnesiana & 2 & 17 & 8.70 & 2.27 \\
\hline & Suma & 23 & 192 & 100 & 26.15 \\
\hline & Prosopis laevigata & 44 & 367 & 86.27 & 50.02 \\
\hline \multirow[t]{3}{*}{ II } & Acacia farnesiana & 7 & 58 & 13.73 & 7.96 \\
\hline & Suma & 51 & 425 & 100 & 57.98 \\
\hline & Prosopis laevigata & 10 & 83 & 71.43 & 11.37 \\
\hline \multirow[t]{2}{*}{ III } & Acacia farnesiana & 4 & 33 & 28.57 & 4.55 \\
\hline & Suma & 14 & 117 & 100 & 15.92 \\
\hline
\end{tabular}




\section{DISCUSIÓN}

Se registraron tres especies pertenecientes a dos familias de plantas vasculares, siendo estos valores bajos con respecto a los reportados por Jiménez et al (2012), quienes en un estudio de regeneración leñosa del matorral espinoso tamaulipeco con historial agrícola con 21 años de abandono registraron 19 especies pertenecientes a 11 familias. La especie más abundante en el área de estudio fue Prosopis laevigata, la cual registró un valor de $84 \%$ (616 $\mathrm{N} \mathrm{ha}^{-1}$ ) del total de los individuos evaluados, seguida de Acacia farnesiana con 14.77\% (108 N ha $^{-1}$ ), sumando un total del $96.18 \%\left(724 \mathrm{~N} \mathrm{ha}^{-1}\right)$. Lo que coincide con los reportado por Estrada et al (2004), Jiménez et al. (2012), Pequeño et al. (2012) y Jiménez et al. (2013), quienes indican que las áreas que han sufrido algún tipo de disturbio por actividades productivas, tienden a presentar una baja disponibilidad de nitrógeno en el suelo, lo que causa el establecimiento de especies con capacidad de fijación de nitrógeno en las primeras fases de sucesión ecológica como Prosopis laveigata y Acacia farnesiana. Al respecto Quiñones et al. (2013) mencionan que Prosopis laveigata enriquece al suelo a su alrededor y propicia la aportación de nutrientes. En la misma ecorregión pero en el sur de Texas, Fulbright et al. (2013) evaluaron una comunidad arbustiva sometida a desmonte 40 años después de un tratamiento de arado (root plowing), comparado con sitios sin disturbio. Donde encontraron que las áreas desmontadas fueron dominadas por Prosopis glandulosa y tuvieron más del cuádruple de densidad en sitios desmontados respecto a los sitios sin disturbio (1 $064 \mathrm{~N} \mathrm{ha}^{-1}, 290 \mathrm{~N} \mathrm{ha}^{-1}$ ), respectivamente. Asimismo, la cobertura aérea de $P$. glandulosa tuvo más del doble de cobertura en sitios desmontados comparado a sitios sin disturbio (58 y $23 \%$ ), respectivamente. Lo que coincide con los resultados registrados en esta investigación donde $P$. glandulosa no solo es la especie mayormente presente en el área, lo que reafirma la capacidad de establecimiento por parte de especies fijadoras de nitrógeno en zonas con disturbio. P. glandulosa es también la especie dominante registrando el porcentaje más alto en cobertura aérea con un $89 \%$.

La dominancia registrada en el área de estudio presenta valores de $5309 \mathrm{~m}^{2} \mathrm{ha}^{-1}$, valores bajos comparados con los valores registrados por Mora et al. (2013), quienes realizaron un estudio sobre la estructura y la composición florística del matorral espinoso tamaulipeco en estadio adulto, y registraron una cobertura de $13973 \mathrm{~m}^{2} \mathrm{ha}^{-1}$. Los bajos valores registrados en la presente investigación son debido a que el área de estudio cuenta con un historial de actividad agrícola y posterior abandono, mientras que la zona evaluada por Mora et al. (2013), es una zona sin historial previo de alguna actividad antropogénica $y$, es por ello que lo tomamos como ecosistema de referencia. La abundancia de individuos por hectárea de acuerdo a las clases diamétricas registradas en el estudio, presentan una línea de tendencia exponencial negativa en la densidad de individuos conforme aumenta el diámetro de los mismos, siendo la clase $0-5 \mathrm{~cm}$ de diámetro la que presenta valores superiores a los $350 \mathrm{~N} \mathrm{ha}^{-1}$. Lo que indica que el sistema se encuentra en fases iniciales de la sucesión secundaria y que existe un estado de regeneración activo, con una gran cantidad de individuos de menor porte y un pequeño número de individuos con diámetros superiores a los $30 \mathrm{~cm}$. Lo que concuerda con Alanís et al. (2008), quienes caracterizaron la regeneración leñosa post-incendio de un ecosistema templado del noreste de México y con Pequeño et al. (2012) quienes realizaron un análisis de la restauración pasiva post-pecuaria en el matorral espinoso tamaulipeco registrando ecosistemas compuestos por individuos con las mismas características fenotípicas, observando mayormente individuos de porte pequeños.

El área evaluada mostró valores de riqueza $\mathrm{D}_{M g}=0.45 \mathrm{y}$ de diversidad alfa de $\mathrm{H}^{\prime}=0.48$, estos valores son bajos comparados con áreas con historial de uso silvícola y agrícola del Matorral Espinoso Tamaulipeco (MET), al respecto Jiménez et al. (2012), registraron valores de riqueza $\mathrm{D}_{M g}$ de $2.17 \mathrm{y}$ de diversidad alfa de H' de 2.27. los cuales son bajos comparados con áreas con sistemas de pastoreo en el MET (Matorral Espinoso Tamaulipeco) evaluados por Molina et al. (2013) quienes reportan valores 
de diversidad alfa $\mathrm{H}^{\prime}=2.22$ y 2.11 . Los valores de riqueza y diversidad obtenidos siguen siendo bajos comparados con los publicados por Pequeño et al. (2012) quienes evaluaron la diversidad de la regeneración de especies leñosas del MET con historial pecuario en el NE de México y obtuvieron valores de riqueza $\mathrm{D}_{M g}=1.40 \mathrm{y}$ de diversidad alfa de $\mathrm{H}^{\prime}=$ 1.27. La baja diversidad de este estudio en comparación a los antes mencionados está directamente asociada a que el resto de los estudios se realizaron en el límite sureño de la distribución del MET, mientras que el presente estudio se desarrolló en una localidad típica del MET. El límite sureño del MET es una zona más favorecedora desde el punto de vista de precipitaciones ya que está más próxima a la Sierra Madre Oriental.

El valor obtenido a partir del índice vertical de especies (A) fue 1.4 con un $A_{\max }$ de 2.2 y un $\mathrm{A}_{\text {rel }}$ de $63.7 \%$, indica diversidad estructural media en los estratos de altura, existiendo dos estratos bien definidos (I y II). Valores de $A_{\text {rel }}$ cercanos a $100 \%$ indican que todas las especies se encuentran distribuidas de forma equitativa en los tres estratos de altura (Mora-Donjuan et al. 2014). Los resultados de la presente investigación muestran similitudes con los presentados por Alanís et al. (2010), Jiménez et al. (2009), Villavicencio et al. (2005) y Mora-Donjuan et al. (2014), donde también determinaron que las comunidades vegetales que evaluaron se presentan casi en su totalidad en dos estratos de altura. En todos los casos los estratos que están bien representados son el II y III (medio y bajo), siendo el I (alto) el que presenta con muy escasas especies (S) y abundancias (N/ha).

\section{CONCLUSIONES}

La comunidad vegetal regenerada después de la actividad agrícola se encuentra conformada por tres especies, siendo Prosopis laevigata y Acacia farnesiana las mayormente presentes con un $96.18 \%$ de abundancia, seguida por Celtis pallida. Se tiene alta presencia de individuos en las clases diamétricas menores, lo que muestra un estado de regeneración activo. El índice de distribución vertical indica que la vegetación presenta una diversidad media en los estratos de altura, registrando un valor de $63.7 \%$ de $\mathrm{A}_{\text {rel }}$.

\section{LITERATURA CITADA}

Alanís E, Jiménez J, Aguirre OA, Treviño E, Jurado E, González MA (2008) Efecto del uso del suelo en la fitodiversidad del matorral espinoso tamaulipeco. Ciencia UANL 11: 56-62.

Alanís E, Jiménez J, Pando M, Aguirre OA, Treviño EJ, Canizales P (2010) Caracterización de la diversidad arbórea en áreas Restauradas post-incendio en el parque ecológico Chipinque, México. Acta Biológica Colombiana 15: 309-324.

Alanís E, Jiménez J, Canizales PA, González H, Mora-Olivo A (2015) Estado actual del conocimiento de la estructura arbórea y arbustiva del matorral espinoso tamaulipeco del noreste de México. Revista lberoamericana de Ciencias 2: 69-80.

Arriaga L (2009) Implicaciones del cambio de uso de suelo en la biodiversidad de los matorrales xerófilos: un enfoque multiescalar. Investigación Ambiental 1: 6-16.

Banco mundial (2016) Datos sobre las cuentas nacionales del Banco Mundial y archivos de datos sobre cuentas nacionales de la OCDE. NW Washington, DC, USA. http://datos.bancomundial.org/indicador/NV.AGR.TOTL. ZS. Fecha de consulta: 28 de abril 2019

Bajaña F, Castillo M, Mosquera G, Segarra P (2014) Elaboración de planes de restauración pasiva para el programa socio bosque región amazónica. Propuesta para definición de áreas prioritarias para restauración, programa socio bosque, documento técnico USAID. Ecuador. 34p 
Corral J, Aguirre O, Jiménez J, Corral S (2005) Un análisis del efecto del aprovechamiento forestal sobre la diversidad estructural en el bosque mesófilo de montaña 'El Cielo', Tamaulipas, México. Investigaciones Agrarias: Sistema de Recursos Forestales 14: 217-228.

Del Río M, Montes F, Cañellas I, Montero G (2003) Índices de diversidad estructural en masas forestales. Investigaciones Agrarias: Sistema de Recursos Forestales 12: 159-176.

Estrada E, Yen AD, Villarreal J (2004) Leguminosas del centro del estado de Nuevo León, México. Anales del Instituto de Biología, Serie Botánica 75: 73-85.

Fulbright TE, Lozano-Cavazos EA, Ruthven III DC, Litt AR (2013) Plant and small vertebrate composition and diversity 36-39 years after rootplowing. Range Ecology and Management 66: 19-25.

Fresco LO (2005) Ciencia y la revolución pecuaria. Revista Enfoques FAO http://www.fao.org/ag/esp/revista/0511 sp1.htm. Fecha de consulta: 28 de abril 2019

García J, Jurado E (2008) Caracterización del matorral con condiciones prístinas en Linares N. L., México. Ra Ximhai 4: 1-21.

Jiménez J, Aguirre O, Kramer H (2001) Análisis de la estructura horizontal y vertical en un ecosistema multicohortal de pino-encino en el norte de México. Investigaciones Agrarias: Sistema de Recursos Forestales 10: 355-366.

Jiménez PJ, Alanís E, Aguirre O, Pando M, González M (2009) Análisis sobre el efecto del uso del suelo en la diversidad estructural del matorral espinoso tamaulipeco. Madera y Bosques 15: 5-20.

Jiménez J, Alanís E, Ruiz J, González M, Yerena J, Alanís G (2012) Diversidad de la regeneración leñosa del matorral espinoso tamaulipeco con historial agrícola en el noreste de México. Ciencia UANL 15: 66-71.

Jiménez J, Alanís E, González MA, Aguirre OA, Treviño EJ (2013) Characterizing woody species regeneration in areas with different land history tenure in the tamaulipan thornscrub, Mexico. The Southwestern Naturalist 58: 299-304.

McMahon M, Valdés A (2011) Análisis del extensionismo Agrícola en México. Organismo para la Cooperación y el Desarrollo Económico. París, Francia. 73p.

Meli P, Holl KD, Benayas JMR, Jones HP, Jones PC, Montoya D, Mateos DM (2017) A global review of past land use, climate, and active vs. passive restoration effects on forest recovery. Plos one 12(2) e0171368. Doi: 10.1371/journal.pone.0171368

Molina, VM, Pando M, Alanís E, Canizales PA, González H, Jiménez J (2013) Composición y diversidad vegetal de dos sistemas de pastoreo en el matorral espinoso tamaulipeco del Noreste de México. Revista Mexicana de Ciencias Pecuarias 4: 361-371.

Mora CA, Alanís E, Jiménez J, González MA, Yerena JI, Cuellar LG (2013) Estructura, composición florística y diversidad del matorral espinoso tamaulipeco, México. Ecología Aplicada 12: 29-34.

Mora-Donjuan CA, Rubio-Camacho EA, Alanís-Rodríguez E, Jiménez-Pérez J, González-Tagle MA, Mata-Balderas JM, Mora-Olivo A (2014) Composición y diversidad vegetal de un área de matorral desértico micrófilo con historial pecuario en el noreste de México. Polibotánica 38: 53-66.

Mostacedo B, Fredericksen TS (2000) Manual de métodos básicos de muestreo y análisis en ecología vegetal. Editora El País, Santa Cruz, Bolivia. 87p.

Pequeño-Ledezma MA, Alanís-Rodríguez E, Jiménez-Pérez J, González-Tagle MA, Yerena-Yamallel JI, CuellarRodríguez G \& Mora-Olivo A (2012) Análisis de la restauración pasiva post-pecuaria en el matorral espinoso tamaulipeco del noreste de México. CienciaUAT 7: 48-53. 
Pequeño-Ledezma M, Alanís-Rodríguez E, Jiménez-Pérez J, Aguirre-Calderón OA, González-Tagle MA \& MolinaGuerra V (2016) Criterios a considerar para desarrollar proyectos de restauración ecológica. Revista Iberoamericana de Ciencias 3: 94-105.

Pretzsch H (2009) Forest Dynamics, Growth and Yield. From Measurement to Model. Springer-Verlag. Berlín Heidelberg, Alemania. 664p

Quiñones G,A, González O,V, Chávez PJR, Vargas MA, Barrientos DF (2013) Evaluación de inoculantes promotores de crecimiento en la producción de plantas de mezquite [Prosopis laevigata (Humb. et Bonpl. ex Willd.) MC Johnst.] en Durango. Revista Mexicana de Ciencias Forestales 4: 42-80.

SAGARPA (2009) Nuevo León: Agenda de innovación agroindustrial. Monterrey, Nuevo León, México. 212 pp. http://www.fupro19.org.mx/wp-content/uploads/2014/01/Agenda-20091.pdf Fecha de consulta: 1 de Octubre 2019

SAGARPA-INEGI (2015) Nota técnica, Encuesta nacional agropecuaria (ENA) 2014. Secretaría de Agricultura, Ganadería, Desarrollo Rural, Pesca y Alimentación - Instituto Nacional de Estadística y Geografía. Aguascalientes, México. 22p.

SEMARNAT (2006) El Medio Ambiente en México 2005: En resumen. Secretaría de Medio Ambiente y Recursos Naturales. México. 91p.

Villavicencio GR, Bauche P, Gallegos A, Santiago AL, Huerta FM (2005) Caracterización estructural y diversidad de comunidades arbóreas de La Sierra de Quila. Boletín IBUG 13: 67-76.

Zahawi RA, Reid JL, Holl KD (2015) Passive restoration can be an effective strategy: a reply to Prach and del Moral. Restoration Ecology 23: 347-348. 